\title{
Scalar and vector perturbations in a universe with nonlinear perfect fluid
}

\author{
Ezgi Canay $^{1, \mathrm{a}}{ }_{\mathbb{1}}$, Ruslan Brilenkov ${ }^{2, \mathrm{~b}}$, Maxim Eingorn ${ }^{3, \mathrm{c}}$, A. Savaş Arapoğlu ${ }^{1, \mathrm{~d}}$, Alexander Zhuk ${ }^{4, \mathrm{e}}$ \\ ${ }^{1}$ Department of Physics, Istanbul Technical University, Maslak, 34469 Istanbul, Turkey \\ ${ }^{2}$ Kapteyn Astronomical Institute, University of Groningen, P.O. Box 800, 9700 AV Groningen, The Netherlands \\ ${ }^{3}$ Department of Mathematics and Physics, North Carolina Central University, 1801 Fayetteville St, Durham, NC 27707, USA \\ ${ }^{4}$ Astronomical Observatory, Odessa I.I. Mechnikov National University, Dvoryanskaya St. 2, Odessa 65082, Ukraine
}

Received: 16 January 2021 / Accepted: 5 March 2021 / Published online: 21 March 2021

(C) The Author(s) 2021

\begin{abstract}
We study a three-component universe filled with dust-like matter in the form of discrete inhomogeneities (e.g., galaxies) and perfect fluids characterized by linear and nonlinear equations of state. Within the cosmic screening approach, we develop the theory of scalar and vector perturbations. None of the energy density contrasts associated with the distinct components is treated as small. Consequently, the derived equations are valid at both sub- and super-horizon scales and enable simulations for a variety of cosmological models.
\end{abstract}

\section{Introduction}

Unraveling the physics behind the accelerated expansion of the late universe is one of the greatest challenges in modern cosmology. Going through a number of models that have been proposed to explain this phenomenon, one may first refer to Einstein's legendary cosmological constant as the underlying energy component. However, though it provides the sought-for behavior, it also brings along the question of what could be the origin of such a constant with the value required for the observed acceleration, yet unanswered. Perfect fluids with the linear equation of state $(\operatorname{EoS}) p=\omega \varepsilon$, $\omega=$ const are also known to drive the acceleration provided that $\omega<-1 / 3$. Nevertheless, $\omega$ should be very close to -1 [1] to be in agreement with observations. Alternatively, there exist the so-called quintessence [2,3] models in which the corresponding source is a scalar field. These include cases

\footnotetext{
a e-mail: ezgicanay@itu.edu.tr (corresponding author)

b e-mail: brilenkov@astro.rug.nl

c e-mail: maxim.eingorn@gmail.com

d e-mail: arapoglu@itu.edu.tr

ee-mail: ai.zhuk2@gmail.com
}

leading to constant $\omega$ [4], shown to impose severe restrictions on the form of the scalar field potential $[5,6]$. Perfect fluids with the nonlinear $\operatorname{EoS} p=f(\varepsilon)$ represent yet another class of models in this direction, and the Chaplygin gas model [7-10] may be studied as an example of those.

As one can see, there exists an extensive zoo of models that can explain the accelerated expansion of the universe. Obviously, the models to survive are those with predictions closest to the observational data. Among such criteria is the observed large-scale structure of the universe described on the basis of the widely studied perturbation theory [11-16]. In this direction, a cosmic screening approach has been proposed in the paper [17] for the scalar and vector perturbations, with the distinctive feature that the gravitational potential satisfies a Helmholtz-type equation and not a Poisson-type one. Consequently, at large cosmological distances from individual sources, the potential undergoes an exponential cutoff. Matter sources in [17] have the form of discrete point-like masses, and it is also important to emphasize that no assumptions are made regarding the smallness of the associated energy density contrast, ensuring the validity of the model both at sub- and super-horizon scales. This approach has been further developed in the papers [18-27]. Particularly in [18,19], generalizations to the case of perfect fluids with the linear $p=\omega \varepsilon$ and nonlinear $p=f(\varepsilon)$ equations of state have been performed, i.e. aside from point-like masses, additional components of two distinct types of perfect fluids have been included in the matter sources. Obviously, the linear component has the background $\operatorname{EoS} \bar{p}=\omega \bar{\varepsilon}$. Nevertheless, for the nonlinear component, it is possible to write $\bar{p}=f(\bar{\varepsilon})$ only in the case of small fluctuations where the expansion $p=f(\bar{\varepsilon})+(\partial f / \partial \varepsilon)_{\bar{\varepsilon}} \delta \varepsilon+(1 / 2)\left(\partial^{2} f / \partial \varepsilon^{2}\right)_{\bar{\varepsilon}} \delta \varepsilon^{2}+\ldots$ works well. This point is disregarded in some papers (see e.g. [28$30])$ whereas in [31-35], for instance, it is clearly stated that the relation $\bar{p}=f(\bar{\varepsilon})$ does not hold in general. 
In [18], the authors considered the case where energy density fluctuations of the nonlinear perfect fluid are small quantities. Meanwhile, density contrasts of the pressureless matter and perfect fluid with linear EoS were set arbitrary. In the present article, we investigate all three matter components with arbitrary density contrasts. Therefore, the considered model applies both to small/astrophysical scales, where matter fluctuations are large, and to large/cosmological distances, where the density contrast is small. We develop the theory of scalar and vector perturbations for this model within the cosmic screening approach and obtain a system of equations which enables the cosmological simulation for arbitrary forms of the function $f(\varepsilon)$.

The paper is structured as follows. In Sect. 2, the basic equations are presented and the theory of scalar and vector perturbations is constructed for the considered model. The main results are summarized in Sect. 3. Appendix is devoted to showing that the auxiliary equations employed in the main proof are satisfied within the adopted accuracy for arbitrary density contrasts.

\section{Scalar and vector perturbations in the cosmic screening approach}

We investigate a universe which contains perfect fluids with nonlinear $\operatorname{EoS} p_{J}=f_{J}\left(\varepsilon_{J}\right), J=1,2, \ldots$ Particular cases include the pressureless perfect fluid with $p=0$ and perfect fluids with linear $\operatorname{EoS} p=\omega \varepsilon, \omega=$ const. The spaceaveraged distribution (denoted by the overbar) of these components determines the dynamics of the homogeneous and isotropic universe described by the Friedmann equation

$\frac{3 \mathscr{H}^{2}}{a^{2}}=\frac{3 H^{2}}{c^{2}}=\kappa \bar{\varepsilon}=\kappa\left(\bar{\varepsilon}_{M}+\sum_{I} \bar{\varepsilon}_{I}+\sum_{J} \bar{\varepsilon}_{J}\right)$,

in which $a(\eta)$ denotes the scale factor, the Hubble parameter $\mathscr{H} \equiv a^{\prime} / a \equiv(d a / d \eta) / a, \eta$ is the conformal time and the constant $\kappa \equiv 8 \pi G_{N} / c^{4}\left(G_{N}\right.$ and $c$ are the Newtonian gravitational constant and the speed of light, respectively). The total averaged energy density $\bar{\varepsilon}$ in the above equation has been split into its constituent parts with respect to their types of EoS: index "M" corresponds to pressureless matter (continuous as well as discrete) and indexes "I" and "J" correspond to perfect fluids with linear and nonlinear EoS, respectively. Fluctuations in the energy densities generate metric perturbations which, in the following, will be studied in terms of their associated scalar and vector components. The perturbed metric in the first-order approximation and in the Poisson gauge reads

$d s^{2}=a^{2}\left[(1+2 \Phi) d \eta^{2}+2 B_{\alpha} d x^{\alpha} d \eta-(1-2 \Phi) \delta_{\alpha \beta} d x^{\alpha} d x^{\beta}\right]$.
The only approximation in our approach is that the metric corrections $\Phi$ and $B_{\alpha}$ as well as the peculiar velocities $\tilde{\mathbf{v}} \equiv\left(\tilde{v}^{1}, \tilde{v}^{2}, \tilde{v}^{3}\right), \tilde{v}^{\alpha} \equiv d x^{\alpha} / d \eta$ are considered small: $\Phi, B_{\alpha}, \tilde{v}^{\alpha} \ll 1$. On the other hand, the smallness of the energy density and pressure fluctuations is not demanded, i.e. the density and pressure contrasts may exceed unity: $\delta \varepsilon / \bar{\varepsilon}$, $\delta p / \bar{p}>1$. This serves as an indicator that our model works both on astrophysical and cosmological scales. Potentials $\Phi$ and $\mathbf{B} \equiv\left(B_{1}, B_{2}, B_{3}\right)$ satisfy the linearized Einstein equations [18]

$$
\begin{aligned}
\triangle \Phi & -3 \mathscr{H}\left(\Phi^{\prime}+\mathscr{H} \Phi\right) \\
= & \frac{1}{2} \kappa a^{2}\left(\delta \varepsilon_{M}+\sum_{I} \delta \varepsilon_{I}+\sum_{J} \delta \varepsilon_{J}\right), \\
\frac{1}{4} & \triangle B_{\alpha}+\frac{\partial}{\partial x^{\alpha}}\left(\Phi^{\prime}+\mathscr{H} \Phi\right) \\
= & \frac{1}{2} \kappa a^{2}\left(-\frac{c^{2}}{a^{3}} \sum_{n} \rho_{n} \tilde{v}_{n}^{\alpha}+\frac{\bar{\rho}_{M} c^{2}}{a^{3}} B_{\alpha}-\sum_{I}\left(\varepsilon_{I}+p_{I}\right) \tilde{v}_{I}^{\alpha}\right. \\
& \left.+\sum_{I}\left(\bar{\varepsilon}_{I}+\bar{p}_{I}\right) B_{\alpha}-\sum_{J}\left(\varepsilon_{J}+p_{J}\right) \tilde{v}_{J}^{\alpha}+\sum_{J}\left(\bar{\varepsilon}_{J}+\bar{p}_{J}\right) B_{\alpha}\right),
\end{aligned}
$$

where $\triangle$ is the Laplace operator in flat comoving space. In the Poisson gauge, the potential $\mathbf{B}$ is subject to the transverse gauge condition $\nabla \mathbf{B} \equiv \delta^{\alpha \beta} \partial B_{\alpha} / \partial x^{\beta}=0$. It should be noted that the indices of three-dimensional vectors are raised and lowered using metric coefficients $\delta_{\alpha \beta}=\delta^{\alpha \beta}$, i.e. there is no difference between covariant and contravariant components.

As mentioned previously, we do not assume the smallness of fluctuations for any type of perfect fluids. Therefore, in contrast to equation (2.9) of [18], where fluctuations of the nonlinear perfect fluid are small quantities, herein we avoid the replacement of the combination $\left(\varepsilon_{J}+p_{J}\right) \tilde{v}_{J}^{\alpha}$ by $\left(\bar{\varepsilon}_{J}+\right.$ $\left.\bar{p}_{J}\right) \tilde{v}_{J}^{\alpha}$. Pressureless matter is taken in the form of discrete point-like masses with comoving mass density

$\rho_{M} \equiv \sum_{n} m_{n} \delta\left(\mathbf{r}-\mathbf{r}_{n}\right) \equiv \sum_{n} \rho_{n}$,

and its averaged energy density $\bar{\varepsilon}_{M}=\bar{\rho}_{M} c^{2} / a^{3}$. For such a component, the energy density fluctuation reads [36-38]

$\delta \varepsilon_{M}=\frac{c^{2}}{a^{3}} \delta \rho_{M}+\frac{3 \bar{\rho}_{M} c^{2}}{a^{3}} \Phi$,

with $\delta \rho_{M} \equiv \rho_{M}-\bar{\rho}_{M}$. This expression should be substituted into the right-hand side (RHS) of Eq. (3). It is worth noting that we have dropped the term $\propto \delta \rho_{M} \Phi$. The point is that $\delta \rho_{M}$ is already a source for the metric correction $\Phi$. Therefore, in the perturbed Einstein equations, the product $\delta \rho_{M} \Phi$ results in corrections of the second order [21]. As for the linear perfect fluid, the energy density can be considered in the form [18-20] 


$$
\begin{aligned}
\varepsilon_{I} & =\frac{A_{I}}{a^{3\left(1+\omega_{I}\right)}}+3\left(1+\omega_{I}\right) \bar{\varepsilon}_{I} \Phi \\
& =\frac{\bar{A}_{I}}{a^{3\left(1+\omega_{I}\right)}}+\frac{\delta A_{I}}{a^{3\left(1+\omega_{I}\right)}}+\frac{3\left(1+\omega_{I}\right) \bar{A}_{I}}{a^{3\left(1+\omega_{I}\right)}} \Phi,
\end{aligned}
$$

where $A_{I} \equiv \bar{A}_{I}+\delta A_{I}+\delta A_{I}$ and $\bar{A}_{I}=$ const. Since each matter component separately satisfies the energy conservation equation (see Eq. (A.20) in [18])

$$
\begin{aligned}
& \varepsilon^{\prime}+3 \mathscr{H}(\varepsilon+p)-3(\varepsilon+p) \Phi^{\prime}+\nabla[(\varepsilon+p) \tilde{\mathbf{v}}]+\nabla[p \mathbf{B}] \\
& \quad=0
\end{aligned}
$$

in which $\varepsilon$ represents any of the individual components and $\Phi$ and $\mathbf{B}$ are the total potentials produced by the combination of components, one can easily show that the function $A_{I}$ fulfills

$A_{I}^{\prime}+\left(1+\omega_{I}\right) \nabla\left(A_{I} \tilde{\mathbf{v}}_{I}\right)=0$

For the averaged quantities, Eq. (8) yields

$\bar{\varepsilon}^{\prime}+3 \mathscr{H}(\bar{\varepsilon}+\bar{p})=0$,

and evidently, $\bar{\varepsilon}_{I}=\bar{A}_{I} / a^{3\left(1+\omega_{I}\right)}$ satisfies this equation.

Let us now turn to the nonlinear perfect fluid with EoS $p_{J}=f_{J}\left(\varepsilon_{J}\right)$, where $f_{J}$ represents some nonlinear function. Since the fluctuations in the energy density and pressure are not restricted to small values, it is no longer possible to substitute $\bar{p}_{J}=f\left(\bar{\varepsilon}_{J}\right)$ for the background pressure; we need to proceed in a rather different way. Similar to Eqs. (6) and (7), we consider the energy density in the form

$\varepsilon_{J}=F_{J}+3\left(\varepsilon_{J}+p_{J}\right) \Phi$,

where $F_{J}$ is an unknown function for which we will derive an equation subsequently. Provided that $|\Phi| \ll 1$, expanding the quantities $\varepsilon_{J}$ and $p_{J}$ accordingly, we may write

$\varepsilon_{J}=F_{J}+3\left[F_{J}+f_{J}\left(F_{J}\right)\right] \Phi$,

$p_{J}=f_{J}\left(F_{J}\right)+\left.3 \frac{\partial f_{J}}{\partial \varepsilon_{J}}\right|_{\varepsilon_{J}=F_{J}}\left[F_{J}+f_{J}\left(F_{J}\right)\right] \Phi$.

It is naturally demanded that the energy density (11) satisfies the conservation equation (8). In this connection, we substitute (12) and (13) into (8), which yields

$$
\begin{aligned}
& {\left[F_{J}{ }^{\prime}+3 \mathscr{H}\left(F_{J}+f_{J}\left(F_{J}\right)\right)\right]\left[1+3 \Phi\left(1+\left.\frac{\partial f_{J}}{\partial \varepsilon_{J}}\right|_{\varepsilon_{J}=F_{J}}\right)\right]} \\
& +\nabla\left[\left(F_{J}+f_{J}\left(F_{J}\right)\right) \tilde{\mathbf{v}}_{J}\right]+\mathbf{B} \nabla f_{J}\left(F_{J}\right)=0 .
\end{aligned}
$$

In obtaining the above expression we have neglected the terms quadratic with respect to $\Phi$ and used the relation $\nabla \mathbf{B}=0$ as well as $f^{\prime}(F)=\partial f /\left.\partial \varepsilon\right|_{\varepsilon=F} F^{\prime}$.
Now, we decompose the functions $F_{J}$ and $f_{J}\left(F_{J}\right)$ into average values and fluctuations as

$F_{J}=\overline{F_{J}}+\delta F_{J}, \quad f_{J}\left(F_{J}\right)=\overline{f_{J}\left(F_{J}\right)}+\delta f_{J}$,

where both quantities $\overline{F_{J}}$ and $\overline{f_{J}\left(F_{J}\right)}$ depend only on time. Obviously, $\bar{\varepsilon}_{J}=\overline{F_{J}}$ and $\bar{p}_{J}=\overline{f_{J}\left(F_{J}\right)}$ and hence the background equation (10) for the " $J$ "-component reads

${\overline{F_{J}}}^{\prime}+3 \mathscr{H}\left(\overline{F_{J}}+\overline{f_{J}\left(F_{J}\right)}\right)=0$.

Substituting the decomposed functions (15) into (14) and taking into account (16), we get

$$
\begin{aligned}
& {\left[\delta F_{J}^{\prime}+3 \mathscr{H}\left(\delta F_{J}+\delta f_{J}\right)\right]} \\
& \quad \times\left[1+3 \Phi\left(1+\left.\frac{\partial f_{J}}{\partial \varepsilon_{J}}\right|_{\varepsilon_{J}=\bar{F}_{J}+\delta F_{J}}\right)\right] \\
& \quad+\nabla\left[\left(F_{J}+f_{J}\left(F_{J}\right)\right) \tilde{\mathbf{v}}_{J}\right]+\mathbf{B} \nabla \delta f_{J}=0 .
\end{aligned}
$$

Before proceeding further, we pause to make a few important comments. First, on large/cosmological scales the quantities $\Phi, B, \tilde{v}$ are of the same order of smallness $\epsilon$, i.e. $\Phi \sim B \sim \tilde{v} \sim \epsilon \ll 1$. Meanwhile at small/astrophysical distances, we have $\Phi \sim \epsilon$ and $B \sim \tilde{v} \Phi$. Second, the perfect fluid is considered to behave the "normal" way. By this we mean that the squared speed of sound $c_{s}^{2}=\delta p / \delta \varepsilon \sim \partial f_{J} / \partial \varepsilon_{J} \lesssim 1$ and, additionally, the ratio of pressure fluctuations over pressure is of the order of its energy density counterpart: $\delta \varepsilon / \varepsilon \sim \delta p / p \Rightarrow \delta \varepsilon_{J} / \varepsilon_{J} \sim \delta f_{J} / f_{J}$. Third, we exploit a useful estimate ${ }^{1}[21,39]$

$\Phi \frac{\delta \varepsilon}{\varepsilon} \sim \tilde{v}^{2}$

Keeping these in mind, the terms multiplied by the scalar perturbation $\Phi$ can be safely neglected in comparison to the rest of the expression in (17). For $\delta f_{J} B \ll f_{J} \tilde{v}_{J}$, we find that the left-hand side (LHS) is further simplified to yield

$\delta F_{J}^{\prime}+3 \mathscr{H}\left(\delta F_{J}+\delta f_{J}\right)+\nabla\left[\left(F_{J}+f_{J}\left(F_{J}\right)\right) \tilde{\mathbf{v}}_{J}\right]=0$,

which, combined with (16), represents the sought-for equation for the function $F_{J}$.

Now, we return to Eqs. (3) and (4) for the potentials $\Phi$ and B. Substituting (7) together with the definitions from (12) and (13) into Eq. (4), we find

\footnotetext{
1 At small scales, $\delta \varepsilon / \varepsilon \sim 1$, thus the peculiar velocities comply with $\tilde{v}^{2} \sim \epsilon$. At large scales, as $\delta \varepsilon / \varepsilon \sim \epsilon$, one gets $\tilde{v} \sim \epsilon$ instead.
} 


$$
\begin{aligned}
\frac{1}{4} \triangle & B_{\alpha}+\frac{\partial}{\partial x^{\alpha}}\left(\Phi^{\prime}+\mathscr{H} \Phi\right) \\
= & \frac{1}{2} \kappa a^{2}\left(-\frac{c^{2}}{a^{3}} \sum_{n} \rho_{n} \tilde{v}_{n}^{\alpha}+\frac{\bar{\rho}_{M} c^{2}}{a^{3}} B_{\alpha}\right. \\
& -\sum_{I} \frac{1+\omega_{I}}{a^{3\left(1+\omega_{I}\right)}} A_{I} \tilde{v}_{I}^{\alpha}+\sum_{I}\left(\bar{\varepsilon}_{I}+\bar{p}_{I}\right) B_{\alpha} \\
& \left.-\sum_{J}\left(F_{J}+f_{J}\left(F_{J}\right)\right) \tilde{v}_{J}^{\alpha}+\sum_{J}\left(\bar{\varepsilon}_{J}+\bar{p}_{J}\right) B_{\alpha}\right),
\end{aligned}
$$

where we have taken into account that the terms proportional to the products $\Phi \tilde{v}_{I, J}^{\alpha}$ are to be neglected in the first order. Moving further, we decompose the terms with $\tilde{v}_{n, I, J}^{\alpha}$ on the RHS into their longitudinal and transverse parts as (see Eqs. (2.24) and (2.25) in [18])

$$
\begin{aligned}
& \sum_{n} \rho_{n} \tilde{\mathbf{v}}_{n}=\nabla \Xi+\left(\sum_{n} \rho_{n} \tilde{\mathbf{v}}_{n}-\nabla \Xi\right), \nabla\left(\sum_{n} \rho_{n} \tilde{\mathbf{v}}_{n}\right)=\Delta \Xi \\
& A_{I} \tilde{\mathbf{v}}_{I}=\nabla \xi_{I}+\left(A_{I} \tilde{\mathbf{v}}_{I}-\nabla \xi_{I}\right), \nabla\left(A_{I} \tilde{\mathbf{v}}_{I}\right)=\Delta \xi_{I} \\
& \left(F_{J}+f_{J}\left(F_{J}\right)\right) \tilde{\mathbf{v}}_{J}=\nabla \zeta_{J}+\left[\left(F_{J}+f_{J}\left(F_{J}\right)\right) \tilde{\mathbf{v}}_{J}-\nabla \zeta_{J}\right] \\
& \nabla\left[\left(F_{J}+f_{J}\left(F_{J}\right)\right) \tilde{\mathbf{v}}_{J}\right]=\Delta \zeta_{J}
\end{aligned}
$$

Here the function $\Xi$ has the form [17]

$$
\Xi=\frac{1}{4 \pi} \sum_{n} m_{n} \frac{\left(\mathbf{r}-\mathbf{r}_{n}\right) \tilde{\mathbf{v}}_{n}}{\left|\mathbf{r}-\mathbf{r}_{n}\right|^{3}}
$$

whereas $\xi_{I}$ and $\zeta_{J}$ are to be determined numerically. Using this system of equations, we split Eq. (20) into scalar and vector parts so as to obtain the equations

$$
\begin{aligned}
& \Phi^{\prime}+\mathscr{H} \Phi=-\frac{\kappa c^{2}}{2 a} \Xi-\frac{\kappa}{2} \sum_{I} \frac{1+\omega_{I}}{a^{1+3 \omega_{I}}} \xi_{I}-\frac{\kappa a^{2}}{2} \sum_{J} \zeta_{J} \\
& \frac{1}{4} \triangle \mathbf{B} \\
&-\left[\frac{\kappa \bar{\rho}_{M} c^{2}}{2 a}+\frac{\kappa a^{2}}{2} \sum_{I}\left(\bar{\varepsilon}_{I}+\bar{p}_{I}\right)+\frac{\kappa a^{2}}{2} \sum_{J}\left(\bar{\varepsilon}_{J}+\bar{p}_{J}\right)\right] \mathbf{B} \\
&=-\frac{\kappa c^{2}}{2 a}\left(\sum_{n} \rho_{n} \tilde{\mathbf{v}}_{n}-\nabla \Xi\right)-\frac{\kappa}{2} \sum_{I} \frac{1+\omega_{I}}{a^{1+3 \omega_{I}}}\left(A_{I} \tilde{\mathbf{v}}_{I}-\nabla \xi_{I}\right) \\
&-\frac{\kappa a^{2}}{2} \sum_{J}\left[\left(F_{J}+f_{J}\left(F_{J}\right)\right) \tilde{\mathbf{v}}_{J}-\nabla \zeta_{J}\right] .
\end{aligned}
$$

Substituting (25) into Eq. (3) and taking into account Eqs. (6), (7) together with the expression

$$
\delta \varepsilon_{J} \equiv \varepsilon_{J}-\bar{\varepsilon}_{J}=\delta F_{J}+3\left(\bar{F}_{J}+\overline{f_{J}\left(F_{J}\right)}\right) \Phi,
$$

we get the equation for the potential $\Phi$ :

$$
\triangle \Phi-\frac{3}{2} \kappa a^{2}\left[\frac{\bar{\rho}_{M} c^{2}}{a^{3}}+\sum_{I} \frac{\left(1+\omega_{I}\right) \bar{A}_{I}}{a^{3\left(1+\omega_{I}\right)}}\right.
$$

$$
\begin{aligned}
& \left.+\sum_{J}\left(\bar{F}_{J}+\overline{f_{J}\left(F_{J}\right)}\right)\right] \Phi \\
= & \frac{1}{2} \kappa a^{2}\left[\frac{c^{2}}{a^{3}} \delta \rho_{M}+\sum_{I} \frac{\delta A_{I}}{a^{3\left(1+\omega_{I}\right)}}+\sum_{J} \delta F_{J}\right] \\
& -\frac{3 \kappa c^{2} \mathscr{H}}{2 a} \Xi-\frac{3 \mathscr{H} \kappa}{2} \sum_{I} \frac{1+\omega_{I}}{a^{1+3 \omega_{I}}} \xi_{I}-\frac{3 \mathscr{H} \kappa a^{2}}{2} \sum_{J} \zeta_{J} .
\end{aligned}
$$

It is possible to reformulate (26) and (28) so that we have

$$
\begin{aligned}
\triangle \Phi & -\frac{a^{2}}{\lambda^{2}} \Phi \\
= & \frac{\kappa c^{2}}{2 a} \delta \rho_{M}+\frac{\kappa a^{2}}{2} \sum_{I} \frac{\delta A_{I}}{a^{3\left(1+\omega_{I}\right)}}+\frac{\kappa a^{2}}{2} \sum_{J} \delta F_{J} \\
& -\frac{3 \kappa c^{2} \mathscr{H}}{2 a} \Xi-\frac{3 \mathscr{H} \kappa}{2} \sum_{I} \frac{1+\omega_{I}}{a^{1+3 \omega_{I}}} \xi_{I}-\frac{3 \mathscr{H} \kappa a^{2}}{2} \sum_{J} \zeta_{J}
\end{aligned}
$$

and

$$
\begin{aligned}
\frac{1}{4} & \triangle \mathbf{B}-\frac{a^{2}}{3 \lambda^{2}} \mathbf{B} \\
= & -\frac{\kappa c^{2}}{2 a}\left(\sum_{n} \rho_{n} \tilde{\mathbf{v}}_{n}-\nabla \Xi\right)-\frac{\kappa}{2} \sum_{I} \frac{1+\omega_{I}}{a^{1+3 \omega_{I}}}\left(A_{I} \tilde{\mathbf{v}}_{I}-\nabla \xi_{I}\right) \\
& -\frac{\kappa a^{2}}{2} \sum_{J}\left[\left(F_{J}+f_{J}\left(F_{J}\right)\right) \tilde{\mathbf{v}}_{J}-\nabla \zeta_{J}\right]
\end{aligned}
$$

where

$$
\begin{aligned}
\lambda \equiv & {\left[\frac{3 \kappa \bar{\rho}_{M} c^{2}}{2 a^{3}}+\frac{3 \kappa}{2} \sum_{I} \frac{\left(1+\omega_{I}\right) \bar{A}_{I}}{a^{3\left(1+\omega_{I}\right)}}\right.} \\
& \left.+\frac{3 \kappa}{2} \sum_{J}\left(\bar{F}_{J}+\overline{f_{J}\left(F_{J}\right)}\right)\right]^{-1 / 2} .
\end{aligned}
$$

With only a single " $J$ "-component ${ }^{2}$ in the universe, Eqs. (29) and (30) are reduced to

$\triangle \Phi-\frac{a^{2}}{\lambda^{2}} \Phi=\frac{\kappa a^{2}}{2} \delta F_{J}-\frac{3 \mathscr{H} \kappa a^{2}}{2} \zeta_{J}$

and

$$
\frac{1}{4} \triangle \mathbf{B}-\frac{a^{2}}{3 \lambda^{2}} \mathbf{B}=-\frac{\kappa a^{2}}{2}\left[\left(F_{J}+f_{J}\left(F_{J}\right)\right) \tilde{\mathbf{v}}_{J}-\nabla \zeta_{J}\right]
$$

respectively, accompanied by the evident redefinition of $\lambda$. It is remarkable that in these equations the scalar and vector perturbations are decoupled. Obviously, we get the same Eq. (29) if we discard the vector perturbations and work from the very beginning in the conformal Newtonian gauge. It is

\footnotetext{
${ }^{2}$ As we have noted above, the " $M$ "- and " $I$ "-components can be considered as particular cases of the " $J$ "-component. For example, for the " $I$ "-component with the EoS $p_{I}=\omega_{I} \varepsilon_{I}$, we have $\partial f_{I} / \partial \varepsilon_{I}=\omega_{I}, F_{I} \equiv$ $A_{I} / a^{3\left(1+\omega_{I}\right)}$ and $A_{I}$ satisfies Eq. (9). Hence, in this particular case Eq. (19) is reduced to (9).
} 
well known that in this gauge the perturbations coincide with the Bardeen gauge-invariant ones.

We also need the momentum conservation equation for the " $J$ "-component. Using (A.23) from [18] and eliminating the terms proportional to $\Phi^{2}, \Phi B, \Phi \tilde{v}, B \tilde{v}$, which are of the higher order of smallness, we obtain

$$
\begin{aligned}
\left(F_{J}\right. & \left.+f_{J}\left(F_{J}\right)\right) \mathbf{B}^{\prime} \\
& +\left[\left(1+\left.\frac{\partial f_{J}}{\partial \varepsilon_{J}}\right|_{\varepsilon_{J}=F_{J}}\right) F_{J}^{\prime}+4 \mathscr{H}\left(F_{J}+f_{J}\left(F_{J}\right)\right)\right] \mathbf{B} \\
& -\left[\left(F_{J}+f_{J}\left(F_{J}\right)\right) \tilde{\mathbf{v}}_{J}-\nabla \zeta_{J}\right]^{\prime} \\
& -4 \mathscr{H}\left[\left(F_{J}+f_{J}\left(F_{J}\right)\right) \tilde{\mathbf{v}}_{J}-\nabla \zeta_{J}\right] \\
& -\nabla\left(\zeta_{J}^{\prime}+4 \mathscr{H}_{J}+f_{J}\left(F_{J}\right)+\left.3 \frac{\partial f_{J}}{\partial \varepsilon_{J}}\right|_{\varepsilon_{J}=F_{J}}\right. \\
& \left.\times\left[F_{J}+f_{J}\left(F_{J}\right)\right] \Phi\right) \\
& -\left(F_{J}+f_{J}\left(F_{J}\right)\right) \nabla \Phi=0 .
\end{aligned}
$$

This equation may readily be used to define the time derivative of peculiar velocity and to this end, one may replace $\mathbf{B}^{\prime}$ with the help of the equation $\mathbf{B}^{\prime}=-2 \mathscr{H} \mathbf{B}[17,18]$.

To conclude this section, we briefly consider the Chaplygin gas model [7-9] as a particular example of the " $J$ "-type nonlinear perfect fluid. The EoS of the modified generalized Chaplygin gas has the form [10]

$p=f(\varepsilon)=\beta \varepsilon-(1+\beta) \frac{A}{\varepsilon^{\alpha}}, \quad \beta, A, \alpha:$ const,

for which the background quantities read

$\bar{\varepsilon}=\bar{F}, \quad \bar{p}=\overline{f(F)}=\beta \bar{F}-A(1+\beta) \overline{\left(\frac{1}{F^{\alpha}}\right)}$.

These satisfy the conservation equation (16)

$\bar{F}^{\prime}+3 \mathscr{H}\left[(1+\beta) \bar{F}-A(1+\beta) \overline{\left(\frac{1}{F^{\alpha}}\right)}\right]=0$,

which serves to define $\bar{F}$. Unfortunately, it cannot be solved analytically since averaging of the unknown function $1 / F^{\alpha}$ is not possible. Therefore, in the case of perfect fluids with nonlinear EoS, models of interest should be investigated numerically. The general strategy is as follows: first, one defines the initial values for $F, \tilde{v}$ and the scale factor $a$. Then, performing iteration, one solves the system of 6 equations which are the energy conservation for the background and perturbed values (16) and (19), the momentum conservation equation (34), the Friedmann equation (1) and equations for the potentials $\Phi$ and B obtained in (32) and (33), respectively. The explicit algorithm for the numerical simulation consists in the following: one starts with solving Eqs. (32) and (33) for $\Phi$ and $\mathbf{B}$ at some moment of time for known sources of these potentials, and then, with the help of their values, determines $F_{J}$ and $\tilde{\mathbf{v}}_{J}$ as well as the scale factor $a$ at the next moment from (16), (19) and (34), as well as (1). After such a straightforward procedure, everything is ready for the new iteration step.

\section{Conclusion}

In our paper we have studied a universe consisting of three types of components. It contains dust-like matter (denoted by the index " $M$ ") in the form of discrete inhomogeneities (e.g., galaxies, galaxy groups and clusters), perfect fluids with linear $\operatorname{EoS} p_{I}=\omega_{I} \varepsilon_{I} \quad\left(\omega_{I}=\right.$ const $)$ and perfect fluids with arbitrary nonlinear EoS $p_{J}=f_{J}\left(\varepsilon_{J}\right)$. The background spacetime geometry is defined by the Friedmann-LemaitreRobertson-Walker metric. All three components have been considered to have arbitrary energy density contrasts. Within the cosmic screening approach, we have developed the theory of scalar and vector perturbations and obtained a system of equations that enables the numerical simulation of models with an arbitrary form of the function $f(\varepsilon)$. Since we have not assumed the smallness of energy density fluctuations, these equations are valid on both small/astrophysical and large/cosmological scales.

Additionally, we have checked some of the important auxiliary equations to demonstrate that they are indeed satisfied (up to the adopted accuracy) for arbitrary density contrasts.

Data Availability Statement This manuscript has no associated data or the data will not be deposited. [Authors' comment: Data sharing is not applicable to this article as no data were generated or analyzed in the study.]

Open Access This article is licensed under a Creative Commons Attribution 4.0 International License, which permits use, sharing, adaptation, distribution and reproduction in any medium or format, as long as you give appropriate credit to the original author(s) and the source, provide a link to the Creative Commons licence, and indicate if changes were made. The images or other third party material in this article are included in the article's Creative Commons licence, unless indicated otherwise in a credit line to the material. If material is not included in the article's Creative Commons licence and your intended use is not permitted by statutory regulation or exceeds the permitted use, you will need to obtain permission directly from the copyright holder. To view a copy of this licence, visit http://creativecomm ons.org/licenses/by/4.0/.

Funded by SCOAP ${ }^{3}$.

\section{Appendix A: Check of the equation (25)}

The cosmic screening approach $[17,18]$ applies to all scales down to astrophysical distances where energy density fluctuations of the perfect fluids are no longer small quantities. In this appendix we intend to demonstrate that Eq. (25) holds for arbitrary values of density contrasts. For this purpose, 
we will be preserving the terms of the type $\delta \rho \Phi, \delta A \Phi, \delta F \Phi$ which can exceed $\bar{\rho} \Phi, \bar{A} \Phi, \bar{F} \Phi$ at small scales.

We have already noted that " $M$ "- and " $I$ "-components can be considered as particular cases of the " $J$ "-component. Hence, it would indeed be sufficient to limit ourselves to the " $J$ "-components in (25). Nevertheless, our reasoning will be clearer if we first prove the equation for the " $M$ "component, then for the "I"-component, and only after that for the " $J$ "-component. Considering the "M"-component separately from the other two is especially justified by the fact that there exist exact expressions for the potentials $\Phi$ and $\mathbf{B}$ in the corresponding model.

\section{Appendix A.1: Pressureless matter}

We consider the " $M$ "--component in the form of discrete point-like masses with the comoving mass density (5). For this case, the potential $\Phi$ in Fourier space reads [17]

$$
\begin{aligned}
\widehat{\Phi}= & -\frac{\kappa c^{2}}{2 a}\left(k^{2}+\frac{3 \kappa \bar{\rho} c^{2}}{2 a}\right)^{-1} \\
& \times\left[\widehat{\delta \rho}+3 i \mathscr{H} \sum_{n} m_{n} e^{-i \mathbf{k} \mathbf{r}_{n}} \frac{\left(\mathbf{k} \tilde{\mathbf{v}}_{n}\right)}{k^{2}}\right],
\end{aligned}
$$

where

$\widehat{\delta \rho}=\sum_{n} \widehat{\rho}_{n}-\widehat{\bar{\rho}}=\sum_{n} m_{n} e^{-i \mathbf{k} \mathbf{r}_{n}}-\bar{\rho}(2 \pi)^{3} \delta(\mathbf{k})$.

Above and hereafter the hat denotes the Fourier-transforms.

In the current arrangement, Eq. (25) takes the form

$\widehat{\Phi}^{\prime}+\mathscr{H} \widehat{\Phi}=-\frac{\kappa c^{2}}{2 a} \widehat{\Xi}$

where [17]

$\widehat{\Xi}=-\frac{i}{k^{2}} \sum_{n} m_{n}\left(\mathbf{k} \tilde{\mathbf{v}}_{n}\right) e^{-i \mathbf{k} \mathbf{r}_{n}}=-\frac{i}{k^{2}} \sum_{n} \widehat{\rho}_{n}\left(\mathbf{k} \tilde{\mathbf{v}}_{n}\right)$.

Differentiating the expression (A.1) with respect to conformal time and neglecting the terms quadratic in $\tilde{\mathbf{v}}_{n}$, we obtain

$$
\begin{aligned}
\widehat{\Phi}^{\prime}= & \frac{\kappa c^{2}}{2 a} \mathscr{H}\left(k^{2}+\frac{3 \kappa \bar{\rho} c^{2}}{2 a}\right)^{-1} \\
& \times\left[\widehat{\delta \rho}+3 i \frac{\mathscr{H}}{a} \sum_{n} m_{n} e^{-i \mathbf{k} \mathbf{r}_{n}} \frac{\left(\mathbf{k} a \tilde{\mathbf{v}}_{n}\right)}{k^{2}}\right] \\
& -\frac{3 \kappa^{2} \bar{\rho} c^{4}}{4 a^{2}} \mathscr{H}\left(k^{2}+\frac{3 \kappa \bar{\rho} c^{2}}{2 a}\right)^{-2} \\
& \times\left[\widehat{\delta \rho}+3 i \frac{\mathscr{H}}{a} \sum_{n} m_{n} e^{-i \mathbf{k r}_{n}} \frac{\left(\mathbf{k} a \tilde{\mathbf{v}}_{n}\right)}{k^{2}}\right]
\end{aligned}
$$

$$
\begin{aligned}
& -\frac{\kappa c^{2}}{2 a}\left(k^{2}+\frac{3 \kappa \bar{\rho} c^{2}}{2 a}\right)^{-1} \\
& \times\left[(\widehat{\delta \rho})^{\prime}+3 i\left(\frac{\mathscr{H}}{a}\right)^{\prime} \sum_{n} m_{n} e^{-i \mathbf{k} \mathbf{r}_{n}} \frac{\left(\mathbf{k} a \tilde{\mathbf{v}}_{n}\right)}{k^{2}}\right. \\
& \left.+3 i \frac{\mathscr{H}}{a} \sum_{n} m_{n} e^{-i \mathbf{k r}_{n}} \frac{\mathbf{k}\left(a \tilde{\mathbf{v}}_{n}\right)^{\prime}}{k^{2}}\right] .
\end{aligned}
$$

Now, taking into account the relation [17]

$$
\begin{aligned}
\sum_{n} \widehat{\rho}_{n}\left(a \tilde{\mathbf{v}}_{n}\right)^{\prime} & =\sum_{n} m_{n} e^{-i \mathbf{k r}_{n}}\left(a \tilde{\mathbf{v}}_{n}\right)^{\prime} \\
& =-a\left[\rho \nabla \Phi \left\lceil+\left[\rho(a \mathbf{B})^{\prime}\right],\right.\right.
\end{aligned}
$$

and expressing the time derivative $(\widehat{\delta \rho})^{\prime}$ using (A.2), we reformulate (A.5) as

$$
\begin{aligned}
\widehat{\Phi}^{\prime}= & -\frac{\kappa c^{2}}{2 a}\left(k^{2}+\frac{3 \kappa \bar{\rho} c^{2}}{2 a}\right)^{-1} \\
& \times\left[-\mathscr{H} \widehat{\delta \rho}-i \mathbf{k} \sum_{n} \hat{\rho}_{n} \tilde{\mathbf{v}}_{n}-3 i \frac{\mathscr{H}^{2}}{a} \sum_{n} \widehat{\rho}_{n} \frac{\left(\mathbf{k} a \tilde{\mathbf{v}}_{n}\right)}{k^{2}}\right. \\
& +3 i\left(\frac{\mathscr{H}}{a}\right)^{\prime} \sum_{n} \widehat{\rho}_{n} \frac{\left(\mathbf{k} a \tilde{\mathbf{v}}_{n}\right)}{k^{2}}+3 \mathscr{H} \bar{\rho} \widehat{\Phi} \\
& \left.-3 i \frac{\mathscr{H}}{k^{2}} \mathbf{k}[\delta \rho \nabla \Phi]^{\wedge}+3 i \frac{\mathscr{H}}{a k^{2}} \mathbf{k}\left[\delta \rho(a \mathbf{B})^{\prime}\right]\right] \\
& -\frac{3 \kappa^{2} \bar{\rho} c^{4}}{4 a^{2}} \mathscr{H}\left(k^{2}+\frac{3 \kappa \bar{\rho} c^{2}}{2 a}\right)^{-2} \\
& \times\left[\widehat{\delta \rho}+3 i \frac{\mathscr{H}}{a} \sum_{n} \hat{\rho}_{n} \frac{\left(\mathbf{k} a \tilde{\mathbf{v}}_{n}\right)}{k^{2}}\right] .
\end{aligned}
$$

The term $-3 i \frac{\mathscr{H}}{k^{2}} \mathbf{k}[\delta \rho \nabla \Phi\lceil\sim \mathscr{H} \delta \rho \cdot \epsilon$ in the square brackets can be neglected in comparison with the term $-\mathscr{H} \widehat{\delta \rho} \sim \mathscr{H} \delta \rho$ (their ratio gives precisely the order of smallness $\epsilon)$. Similarly, the term $3 i \frac{\mathscr{H}}{a k^{2}} \mathbf{k}\left[\delta \rho(a \mathbf{B})^{\prime}\right]$ is much less than the term $-3 i \frac{\mathscr{H}^{2}}{a} \sum_{n} \widehat{\rho}_{n} \frac{\left(\mathbf{k} a \tilde{\mathbf{v}}_{n}\right)}{k^{2}}$. Their ratio is of the order of $\delta \rho B /(\rho \tilde{v}) \sim B \tilde{v} /\left(\rho \tilde{v}^{2} / \delta \rho\right) \sim B \tilde{v} / \Phi \sim \epsilon$. Here, we use the relation $\mathbf{B}^{\prime}=-2 \mathscr{H} \mathbf{B}$, the estimate (18) and take into account that at small/astrophysical scales $B \sim \tilde{v} \Phi$ while at large/cosmological scales $B \sim \Phi$ (see also footnote 1).

Substituting the expressions for $\widehat{\Phi}$ and $\widehat{\Phi}^{\prime}$ into the LHS of (A.3), we get

$$
\begin{aligned}
\widehat{\Phi}^{\prime} & +\mathscr{H} \widehat{\Phi}=-\frac{\kappa c^{2}}{2 a}\left(k^{2}+\frac{3 \kappa \bar{\rho} c^{2}}{2 a}\right)^{-1} \\
& \times\left[-i \sum_{n} \widehat{\rho}_{n}\left(\mathbf{k} \tilde{\mathbf{v}}_{n}\right)+3 i\left(\frac{\mathscr{H}}{a}\right)^{\prime} \sum_{n} \widehat{\rho}_{n} \frac{\left(\mathbf{k} a \tilde{\mathbf{v}}_{n}\right)}{k^{2}}\right] \\
& +\frac{3 \kappa^{2} \bar{\rho} c^{4}}{4 a^{2}} \mathscr{H}\left(k^{2}+\frac{3 \kappa \bar{\rho} c^{2}}{2 a}\right)^{-2}
\end{aligned}
$$




$$
\begin{aligned}
& \times\left[\widehat{\delta \rho}+3 i \mathscr{H} \sum_{n} m_{n} e^{-i \mathbf{k} \mathbf{r}_{n}} \frac{\left(\mathbf{k} \tilde{\mathbf{v}}_{n}\right)}{k^{2}}\right] \\
& -\frac{3 \kappa^{2} \bar{\rho} c^{4}}{4 a^{2}} \mathscr{H}\left(k^{2}+\frac{3 \kappa \bar{\rho} c^{2}}{2 a}\right)^{-2} \\
& \times\left[\widehat{\delta \rho}+3 i \frac{\mathscr{H}}{a} \sum_{n} \widehat{\rho}_{n} \frac{\left(\mathbf{k} a \tilde{\mathbf{v}}_{n}\right)}{k^{2}}\right] \\
= & \frac{\kappa c^{2}}{2 a^{2}} i\left(k^{2}+\frac{3 \kappa \bar{\rho} c^{2}}{2 a}\right)^{-1}\left[k^{2}-3 a\left(\frac{\mathscr{H}}{a}\right)^{\prime}\right] \sum_{n} \widehat{\rho}_{n} \frac{\left(\mathbf{k} a \tilde{\mathbf{v}}_{n}\right)}{k^{2}} \\
= & \frac{\kappa c^{2}}{2 a^{2}} i\left(k^{2}+\frac{3 \kappa \bar{\rho} c^{2}}{2 a}\right)^{-1} \\
& \times\left[k^{2}+3\left(\mathscr{H}{ }^{2}-\mathscr{H}{ }^{\prime}\right)\right] \sum_{n} \widehat{\rho}_{n} \frac{\left(\mathbf{k} a \tilde{\mathbf{v}}_{n}\right)}{k^{2}} \\
= & \frac{\kappa c^{2}}{2 a^{2}} i\left(k^{2}+\frac{3 \kappa \bar{\rho} c^{2}}{2 a}\right)^{-1}\left(k^{2}+\frac{3 \kappa \bar{\rho} c^{2}}{2 a}\right) \sum_{n} \widehat{\rho}_{n} \frac{\left(\mathbf{k} a \tilde{\mathbf{v}}_{n}\right)}{k^{2}} \\
= & \frac{\kappa c^{2}}{2 a} i \sum_{n} \widehat{\rho}_{n} \frac{\left(\mathbf{k} \tilde{\mathbf{v}}_{n}\right)}{k^{2}},
\end{aligned}
$$

and this exactly coincides with the RHS of (A.3) (see Eq. (A.4)).

Appendix A.2: Perfect fluid with linear equation of state

In this section we consider perfect fluids with linear EoS $p_{I}=\omega_{I} \varepsilon_{I}, \omega_{I}=$ const. From Eq. (25) we obtain

$\triangle \Phi^{\prime}+\mathscr{H} \triangle \Phi=-\frac{1}{2} \kappa a^{2} \sum_{I}\left(1+\omega_{I}\right) \Delta \zeta_{I}$,

where

$\zeta_{I} \equiv \frac{\xi_{I}}{a^{3\left(1+\omega_{I}\right)}}$.

Our intention now is to prove that Eq. (A.9) holds for arbitrary values of energy density fluctuations.

From Eq. (29) and taking into account the definition (31) for $\lambda$, we get

$$
\begin{aligned}
\Delta \Phi= & \frac{3}{2} \kappa a^{2}\left[\sum_{I} \frac{1+\omega_{I}}{a^{3\left(1+\omega_{I}\right)}} \bar{A}_{I}\right] \Phi \\
& +\frac{1}{2} \kappa a^{2} \sum_{I} \frac{\delta A_{I}}{a^{3\left(1+\omega_{I}\right)}}-\frac{3}{2} \kappa a^{2} \mathscr{H} \sum_{I}\left(1+\omega_{I}\right) \zeta_{I} .
\end{aligned}
$$

The time derivative of this equation is

$$
\begin{aligned}
\Delta \Phi^{\prime}= & \frac{3}{2} \kappa a^{2}\left[\sum_{I} \frac{1+\omega_{I}}{a^{3\left(1+\omega_{I}\right)}} \bar{A}_{I}\right]\left(\Phi^{\prime}+2 \mathscr{H} \Phi\right) \\
& -\frac{9}{2} \kappa a^{2} \mathscr{H}\left[\sum_{I} \frac{\left(1+\omega_{I}\right)^{2}}{a^{3\left(1+\omega_{I}\right)}} \bar{A}_{I}\right] \Phi \\
& -\frac{1}{2} \kappa a^{2} \mathscr{H} \sum_{I} \frac{\delta A_{I}}{a^{3\left(1+\omega_{I}\right)}}+\frac{1}{2} \kappa a^{2} \sum_{I} \frac{\delta A_{I}^{\prime}}{a^{3\left(1+\omega_{I}\right)}}
\end{aligned}
$$

$$
\begin{aligned}
& -\frac{3}{2} \kappa a^{2} \mathscr{H} \sum_{I} \omega_{I} \frac{\delta A_{I}}{a^{3\left(1+\omega_{I}\right)}} \\
& -\frac{3}{2} \kappa a^{2}\left(2 \mathscr{H}^{2}+\mathscr{H}^{\prime}\right) \sum_{I}\left(1+\omega_{I}\right) \zeta_{I} \\
& -\frac{3}{2} \kappa a^{2} \mathscr{H} \sum_{I}\left(1+\omega_{I}\right) \zeta_{I}^{\prime},
\end{aligned}
$$

and substitution of (A.11) and (A.12) into the LHS of (A.9) results in

$$
\begin{aligned}
& \frac{3}{2} \kappa a^{2}\left[\sum_{I} \frac{1+\omega_{I}}{a^{3\left(1+\omega_{I}\right)}} \bar{A}_{I}\right]\left(\Phi^{\prime}+3 \mathscr{H} \Phi\right) \\
& -\frac{9}{2} \kappa a^{2} \mathscr{H}\left[\sum_{I} \frac{\left(1+\omega_{I}\right)^{2}}{a^{3\left(1+\omega_{I}\right)}} \bar{A}_{I}\right] \Phi+\frac{1}{2} \kappa a^{2} \sum_{I} \frac{\delta A_{I}^{\prime}}{a^{3\left(1+\omega_{I}\right)}} \\
& -\frac{3}{2} \kappa a^{2} \mathscr{H} \sum_{I} \omega_{I} \frac{\delta A_{I}}{a^{3\left(1+\omega_{I}\right)}} \\
& -\frac{3}{2} \kappa a^{2}\left(3 \mathscr{H}^{2}+\mathscr{H}^{\prime}\right) \sum_{I}\left(1+\omega_{I}\right) \zeta_{I} \\
& -\frac{3}{2} \kappa a^{2} \mathscr{H} \sum_{I}\left(1+\omega_{I}\right) \zeta_{I}^{\prime} \\
& =-\frac{1}{2} \kappa a^{2} \sum_{I}\left(1+\omega_{I}\right) \triangle \zeta_{I},
\end{aligned}
$$

where the terms $\propto \kappa a^{2} \mathscr{H} \delta A_{I} / a^{3\left(1+\omega_{I}\right)}$ have been cancelled.

In order to proceed, we need to determine the quantities $\delta A_{I}^{\prime}$ and $\zeta_{I}^{\prime}$. For this purpose, we employ (7) as the energy density $\varepsilon_{I}=\bar{\varepsilon}_{I}+\delta \varepsilon_{I}$ of the " $I$ "-component and re-express Eq. (8) in terms of this component only, which reads

$$
\begin{aligned}
& \frac{\delta A_{I}^{\prime}}{a^{3\left(1+\omega_{I}\right)}}-3\left(1+\omega_{I}\right) \frac{\delta A_{I}}{a^{3\left(1+\omega_{I}\right)}} \Phi^{\prime}+\left(1+\omega_{I}\right) \triangle \zeta_{I} \\
& \quad+\nabla\left[\delta p_{I} \mathbf{B}\right]=0 .
\end{aligned}
$$

Here we have taken into account the definition (22) as well as the transverse gauge condition $\nabla \mathbf{B}=0$. It is worth noting that neither of the cases $\left|\omega_{I}\right| \gg 1$ or $\left|1+\omega_{I}\right| \ll 1$ are being considered in the current configuration. Now, the second term in (A.14) can be neglected in comparison with the first one, since $\delta A_{I} \Phi^{\prime}=\left(\delta A_{I} \Phi\right)^{\prime}-\delta A_{I}^{\prime} \Phi \ll \delta A_{I}^{\prime}$ :

$\frac{\delta A_{I}^{\prime}}{a^{3\left(1+\omega_{I}\right)}}=-\left(1+\omega_{I}\right) \triangle \zeta_{I}-\frac{\omega_{I}}{a^{3\left(1+\omega_{I}\right)}} \nabla\left[\delta A_{I} \mathbf{B}\right]$.

From $\Delta \zeta_{I}=\nabla\left(A_{I} \tilde{\mathbf{v}}_{I} / a^{3\left(1+\omega_{I}\right)}\right)$, it is possible to further conclude that the second term on the RHS may again be neglected in comparison with the first one, if we also take into account the inequality $\left(\delta A_{I} / A_{I}\right) B \ll \tilde{v}_{I}$ (similar to the ones used in previous sections). Consequently,

$\frac{\delta A_{I}^{\prime}}{a^{3\left(1+\omega_{I}\right)}}=-\left(1+\omega_{I}\right) \triangle \zeta_{I}$. 
Further on, in order to find $\zeta_{I}^{\prime}$, we use the momentum conservation equation (A.23) in [18] applied to the " $I$ "component (which is the analogue of (34) for the " $J$ "component):

$$
\begin{gathered}
\left(1+\omega_{I}\right)\left[\varepsilon_{I} \mathbf{B}\right]^{\prime}-\left(1+\omega_{I}\right)\left[\varepsilon_{I} \tilde{\mathbf{v}}_{I}\right]^{\prime}+4 \mathscr{H}\left(1+\omega_{I}\right) \varepsilon_{I} \mathbf{B} \\
-4 \mathscr{H}\left(1+\omega_{I}\right) \varepsilon_{I} \tilde{\mathbf{v}}_{I}-\omega_{I} \nabla \varepsilon_{I}-\left(1+\omega_{I}\right) \varepsilon_{I} \nabla \Phi=0 .
\end{gathered}
$$

Using the definition (7), eliminating the second order terms, replacing $\mathbf{B}^{\prime}$ with the expression $-2 \mathscr{H} \mathbf{B}$ and then acting by $\nabla$, we obtain

$$
\begin{aligned}
& \frac{\left(1+\omega_{I}\right)}{a^{3\left(1+\omega_{I}\right)}} \nabla\left[\delta A_{I}^{\prime} \mathbf{B}\right]-\frac{3\left(1+\omega_{I}\right)^{2}}{a^{3\left(1+\omega_{I}\right)}} \mathscr{H} \nabla\left[\delta A_{I} \mathbf{B}\right] \\
& +2 \mathscr{H} \frac{\left(1+\omega_{I}\right)}{a^{3\left(1+\omega_{I}\right)}} \nabla\left[\delta A_{I} \mathbf{B}\right] \\
& -\left(1+\omega_{I}\right) \triangle \zeta_{I}^{\prime}-4 \mathscr{H}\left(1+\omega_{I}\right) \triangle \zeta_{I} \\
& -\frac{\omega_{I}}{a^{3\left(1+\omega_{I}\right)}} \Delta \delta A_{I}-\frac{3 \omega_{I}\left(1+\omega_{I}\right) \bar{A}_{I}}{a^{3\left(1+\omega_{I}\right)}} \triangle \Phi \\
& -\frac{\left(1+\omega_{I}\right) \bar{A}_{I}}{a^{3\left(1+\omega_{I}\right)}} \Delta \Phi-\frac{\left(1+\omega_{I}\right)}{a^{3\left(1+\omega_{I}\right)}} \nabla\left[\delta A_{I} \nabla \Phi\right]=0 .
\end{aligned}
$$

Since $\Delta \zeta_{I}=\nabla\left(A_{I} \tilde{\mathbf{v}}_{I} / a^{3\left(1+\omega_{I}\right)}\right)$, all terms in the first line can be neglected in comparison with the first and second terms in the second line (the ratios reduce to $\sim$ $\left.\left(\delta A_{I} / A_{I}\right)\left(B / \tilde{v}_{I}\right) \sim B \tilde{v}_{I} / \Phi\right)$. Hence,

$$
\begin{gathered}
-\left(1+\omega_{I}\right) \triangle \zeta_{I}^{\prime}-4 \mathscr{H}\left(1+\omega_{I}\right) \triangle \zeta_{I}-\frac{\omega_{I}}{a^{3\left(1+\omega_{I}\right)}} \Delta \delta A_{I} \\
-\frac{\left(1+3 \omega_{I}\right)\left(1+\omega_{I}\right) \bar{A}_{I}}{a^{3\left(1+\omega_{I}\right)}} \Delta \Phi \\
-\frac{\left(1+\omega_{I}\right)}{a^{3\left(1+\omega_{I}\right)}} \nabla\left[\delta A_{I} \nabla \Phi\right]=0,
\end{gathered}
$$

and we arrive at

$$
\begin{aligned}
\left(1+\omega_{I}\right) \zeta_{I}^{\prime}= & -4 \mathscr{H}\left(1+\omega_{I}\right) \zeta_{I}-\frac{\omega_{I}}{a^{3\left(1+\omega_{I}\right)}} \delta A_{I} \\
& -\frac{\left(1+3 \omega_{I}\right)\left(1+\omega_{I}\right) \bar{A}_{I}}{a^{3\left(1+\omega_{I}\right)}} \Phi \\
& -\frac{\left(1+\omega_{I}\right)}{a^{3\left(1+\omega_{I}\right)}} \Delta^{-1} \nabla\left[\delta A_{I} \nabla \Phi\right] .
\end{aligned}
$$

Substituting Eqs. (25) (for the "I"-component), (A.16) and (A.20) (for $\Phi^{\prime}, \delta A_{I}^{\prime}$, and $\zeta_{I}^{\prime}$, respectively) into Eq. (A.13) and simplifying, which amounts to cancelling the terms $\propto$ $\kappa a^{2} \mathscr{H} \sum_{I} \omega_{I} \delta A_{I} / a^{3\left(1+\omega_{I}\right)}$, we get

$$
\begin{aligned}
\frac{3}{2} \kappa a^{2} & {\left[\sum_{I} \frac{1+\omega_{I}}{a^{3\left(1+\omega_{I}\right)}} \bar{A}_{I}\right]\left(2 \mathscr{H} \Phi-\frac{1}{2} \kappa a^{2} \sum_{I}\left(1+\omega_{I}\right) \zeta_{I}\right) } \\
- & \frac{9}{2} \kappa a^{2} \mathscr{H}\left[\sum_{I} \frac{\left(1+\omega_{I}\right)^{2}}{a^{3\left(1+\omega_{I}\right)}} \bar{A}_{I}\right] \Phi
\end{aligned}
$$

$$
\begin{aligned}
& -\frac{3}{2} \kappa a^{2}\left(\mathscr{H}^{\prime}-\mathscr{H}^{2}\right) \sum_{I}\left(1+\omega_{I}\right) \zeta_{I} \\
& +\frac{3}{2} \kappa a^{2} \mathscr{H} \sum_{I}\left[\frac{\left(1+3 \omega_{I}\right)\left(1+\omega_{I}\right)}{a^{3\left(1+\omega_{I}\right)}} \bar{A}_{I}\right] \Phi \\
& +\frac{3}{2} \kappa a^{2} \mathscr{H} \Delta^{-1}\left[\sum_{I} \frac{\left(1+\omega_{I}\right)}{a^{3\left(1+\omega_{I}\right)}} \nabla\left[\delta A_{I} \nabla \Phi\right]\right]=0 .
\end{aligned}
$$

Here, the third line represents a sum of summands of the order $\sim \delta A_{I} \Phi$ (with factor $\sim\left(1+\omega_{I}\right)$ ), and each summand of this order can be ignored in view of the fact that the terms of the superior order $\sim \delta A_{I}$ (with factors $\sim 1$ (see Eq. (A.13)) and $\sim \omega_{I}$ ) have already been cancelled in the previous steps. Our reasoning is that since we keep the terms in our equations up to only a certain order of smallness, previous cancellations of terms do not correspond to identical zero. To respect the adopted accuracy, it is necessary to discard also the terms with higher orders of smallness than these. Therefore, from (A.21) we obtain

$$
\begin{aligned}
\frac{1}{2} \kappa a^{2} & {\left[\sum_{I} \frac{1+\omega_{I}}{a^{3\left(1+\omega_{I}\right)}} \bar{A}_{I}\right] \sum_{I}\left(1+\omega_{I}\right) \zeta_{I} } \\
+ & \left(\mathscr{H}^{\prime}-\mathscr{H}^{2}\right) \sum_{I}\left(1+\omega_{I}\right) \zeta_{I}=0
\end{aligned}
$$

and this is an identity. Hence, Eq. (A.9) proves adequate within the considered accuracy.

Appendix A.3: Perfect fluid with nonlinear equation of state

Let us now turn to the nonlinear perfect fluid with $\operatorname{EoS} p_{J}=$ $f_{J}\left(\varepsilon_{J}\right)$ where $f_{J}$ is a nonlinear function. In this case, Eq. (25) reads

$\Phi^{\prime}+\mathscr{H} \Phi=-\frac{\kappa a^{2}}{2} \sum_{J} \zeta_{J}$,

and acting on it with the Laplace operator, we get

$\triangle \Phi^{\prime}+\mathscr{H} \triangle \Phi=-\frac{\kappa a^{2}}{2} \sum_{J} \triangle \zeta_{J}$.

$\triangle \Phi$ can be expressed with the help of Eq. (32). Differentiating it with respect to the conformal time $\eta$, we find

$$
\begin{aligned}
\triangle \Phi^{\prime}= & \frac{3 \kappa a^{2}}{2} \sum_{J}\left(\bar{F}_{J}+\overline{f_{J}\left(F_{J}\right)}\right) \Phi^{\prime} \\
& -\frac{3 \kappa a^{2} \mathscr{H}}{2} \sum_{J}\left(\bar{F}_{J}+\overline{f_{J}\left(F_{J}\right)}\right) \Phi \\
& +\frac{3 \kappa a^{2}}{2} \sum_{J}{\overline{f_{J}\left(F_{J}\right)}}^{\prime} \Phi
\end{aligned}
$$




$$
\begin{gathered}
+\kappa a^{2} \mathscr{H} \sum_{J} \delta F_{J}+\frac{\kappa a^{2}}{2} \sum_{J} \delta F_{J}^{\prime} \\
-\frac{3 \kappa a^{2}}{2}\left(\mathscr{H}^{\prime}+2 \mathscr{H}^{2}\right) \sum_{J} \zeta_{J} \\
-\frac{3 \kappa a^{2} \mathscr{H}}{2} \sum_{J} \zeta_{J}^{\prime} .
\end{gathered}
$$

Then, substituting $\triangle \Phi^{\prime}$ (from Eq. (A.25)), $\triangle \Phi$ (from Eq. (32)) and $\Phi^{\prime}$ (from (A.23)) into Eq. (A.24), we obtain

$$
\begin{aligned}
& \frac{3 \kappa a^{2} \mathscr{H}}{2} \sum_{J}\left(\overline{F_{J}+\overline{f_{J}\left(F_{J}\right)}}\right) \Phi \\
& -\frac{3 \kappa a^{2}}{2} \sum_{J}\left(\overline{\left.\frac{\partial f_{J}}{\partial \varepsilon_{J}}\right|_{\varepsilon_{J}=F_{J}} F_{J}^{\prime}}\right) \Phi-\frac{3 \kappa a^{2} \mathscr{H}}{2} \sum_{J} \delta F_{J} \\
& -\frac{\kappa a^{2}}{2} \sum_{J} \delta F_{J}^{\prime}+\frac{3 \kappa a^{2}}{2} 4 \mathscr{H}^{2} \sum_{J} \zeta_{J}+\frac{3 \kappa a^{2} \mathscr{H}}{2} \sum_{J} \zeta_{J}^{\prime} \\
& =\frac{\kappa a^{2}}{2} \sum_{J} \Delta \zeta_{J},
\end{aligned}
$$

where we have also employed the equation $\kappa a^{2} \sum_{J}\left(\bar{\varepsilon}_{J}+\right.$ $\left.\bar{p}_{J}\right)=2\left(\mathscr{H}^{2}-\mathscr{H}^{\prime}\right)$.

To determine the expression for $\zeta_{J}^{\prime}$, we now take the gradient of (34), which yields

$$
\begin{aligned}
& \mathbf{B}^{\prime} \nabla\left(F_{J}+f_{J}\left(F_{J}\right)\right)+\mathbf{B}\left[\left(1+\left.\frac{\partial f_{J}}{\partial \varepsilon_{J}}\right|_{\varepsilon_{J}=F_{J}}\right) \nabla F_{J}^{\prime}\right. \\
& \left.+F_{J}^{\prime} \nabla\left(\left.\frac{\partial f_{J}}{\partial \varepsilon_{J}}\right|_{\varepsilon_{J}=F_{J}}\right)+4 \mathscr{H} \nabla\left(F_{J}+f_{J}\left(F_{J}\right)\right)\right] \\
& -\Delta\left(\zeta_{J}^{\prime}+4 \mathscr{H}_{J}+f_{J}\left(F_{J}\right)\right. \\
& \left.-\left.3 \frac{\partial f_{J}}{\partial \varepsilon_{J}}\right|_{\varepsilon_{J}=F_{J}}\left[F_{J}+f_{J}\left(F_{J}\right)\right] \Phi\right) \\
& -\nabla\left(F_{J}+f_{J}\left(F_{J}\right)\right) \nabla \Phi-\left(F_{J}+f_{J}\left(F_{J}\right)\right) \triangle \Phi=0,
\end{aligned}
$$

and acting on with the inverse Laplacian, we obtain

$$
\begin{aligned}
\zeta_{J}^{\prime}= & -4 \mathscr{H} \zeta_{J}-f_{J}\left(F_{J}\right)-\left.3 \frac{\partial f_{J}}{\partial \varepsilon_{J}}\right|_{\varepsilon_{J}=F_{J}}\left[F_{J}+f_{J}\left(F_{J}\right)\right] \Phi \\
& +\Delta^{-1}\left[\mathbf{B}^{\prime} \nabla\left(F_{J}+f_{J}\left(F_{J}\right)\right)+\mathbf{B}\left(1+\left.\frac{\partial f_{J}}{\partial \varepsilon_{J}}\right|_{\varepsilon_{J}=F_{J}}\right) \nabla F_{J}^{\prime}\right. \\
& \left.+\mathbf{B} F_{J}^{\prime} \nabla\left(\left.\frac{\partial f_{J}}{\partial \varepsilon_{J}}\right|_{\varepsilon_{J}=F_{J}}\right)+4 \mathscr{H} \mathbf{B} \nabla\left(F_{J}+f_{J}\left(F_{J}\right)\right)\right] \\
& -\triangle^{-1}\left[\nabla\left(F_{J}+f_{J}\left(F_{J}\right)\right) \nabla \Phi+\left(F_{J}+f_{J}\left(F_{J}\right)\right) \triangle \Phi\right] .
\end{aligned}
$$

Substituting $\zeta_{J}^{\prime}$ and $\delta F_{J}^{\prime}$ (from Eq. (19)) into (A.26), after lengthy but simple algebra, we find

$$
\begin{aligned}
& \frac{3 \kappa a^{2}}{2} \sum_{J}\left(\overline{\left.\frac{\partial f_{J}}{\partial \varepsilon_{J}}\right|_{\varepsilon_{J}=F_{J}} F_{J}^{\prime}}\right) \Phi \\
& +\frac{3 \kappa a^{2} \mathscr{H}}{2} \sum_{J}\left[\overline{f_{J}\left(F_{J}\right)}+\left.3 \frac{\partial f_{J}}{\partial \varepsilon_{J}}\right|_{\varepsilon_{J}=F_{J}}\left[F_{J}+f_{J}\left(F_{J}\right)\right] \Phi\right] \\
& \quad-\frac{3 \kappa a^{2} \mathscr{H}}{2} \sum_{J} \Delta^{-1}\left[2 \mathscr{H} \mathbf{B} \nabla\left(\delta F_{J}+\delta f_{J}\right)\right. \\
& \left.+\mathbf{B}\left(1+\left.\frac{\partial f_{J}}{\partial \varepsilon_{J}}\right|_{\varepsilon_{J}=F_{J}}\right) \nabla \delta F_{J}^{\prime}+\mathbf{B} F_{J}^{\prime} \nabla\left(\left.\frac{\partial f_{J}}{\partial \varepsilon_{J}}\right|_{\varepsilon_{J}=F_{J}}\right)\right] \\
& +\frac{3 \kappa a^{2} \mathscr{H}}{2} \sum_{J} \Delta^{-1} \nabla\left[\left(\delta F_{J}+\delta f_{J}\right) \nabla \Phi\right]=0,
\end{aligned}
$$

where the terms $\propto \kappa a^{2} \mathscr{H}^{2} \sum_{J} \zeta_{J}, \propto \kappa a^{2} \mathscr{H} \sum_{J}\left(\delta F_{J}+\delta f_{J}\right)$, $\propto \kappa a^{2} \sum_{J} \triangle \zeta_{J}, \propto \kappa a^{2} \mathscr{H} \sum_{J}\left(\bar{F}_{J}+\overline{f_{J}\left(F_{J}\right)}\right) \Phi$ have been cancelled. Back in the representation without $\triangle^{-1}$ (i.e. acting on by $\triangle$ ), this equation reads

$$
\begin{aligned}
& \frac{3 \kappa a^{2}}{2} \sum_{J}\left(\overline{\left.\frac{\partial f_{J}}{\partial \varepsilon_{J}}\right|_{\varepsilon_{J}=F_{J}} F_{J}^{\prime}}\right) \triangle \Phi \\
& +\frac{3 \kappa a^{2} \mathscr{H}}{2} \sum_{J} \Delta\left[\left.3 \frac{\partial f_{J}}{\partial \varepsilon_{J}}\right|_{\varepsilon_{J}=F_{J}}\left[F_{J}+f_{J}\left(F_{J}\right)\right] \Phi\right] \\
& -\frac{3 \kappa a^{2} \mathscr{H}}{2} \sum_{J}\left[2 \mathscr{H} \mathbf{B} \nabla\left(\delta F_{J}+\delta f_{J}\right)\right. \\
& +\mathbf{B}\left[\left(1+\left.\frac{\partial f_{J}}{\partial \varepsilon_{J}}\right|_{\varepsilon_{J}=F_{J}}\right) \nabla \delta F_{J}^{\prime}\right. \\
& \left.\left.+F_{J}^{\prime} \nabla\left(\left.\frac{\partial f_{J}}{\partial \varepsilon_{J}}\right|_{\varepsilon_{J}=F_{J}}\right)\right]\right] \\
& +\frac{3 \kappa a^{2} \mathscr{H}}{2} \sum_{J} \nabla\left[\left(\delta F_{J}+\delta f_{J}\right) \nabla \Phi\right]=0 .
\end{aligned}
$$

The second term in the first line (i.e. the term in large square brackets) can be expressed with the help of Eq. (14). Then, using Eqs. (16) and (19), eliminating the second order terms and exploiting the condition $|\Phi| \ll 1$, we arrive at

$$
\begin{aligned}
\sum_{J} & \left(\overline{\left.\frac{\partial f_{J}}{\partial \varepsilon_{J}}\right|_{\varepsilon_{J}=F_{J}} F_{J}^{\prime}}\right) \Delta \Phi-\sum_{J} \Delta\left[\left.\frac{\partial f_{J}}{\partial \varepsilon_{J}}\right|_{\varepsilon_{J}=F_{J}} \bar{F}_{J}^{\prime} \Phi\right] \\
& -\mathscr{H} \sum_{J}\left[2 \mathscr{H} \mathbf{B} \nabla\left(\delta F_{J}+\delta f_{J}\right)\right. \\
& \left.+\mathbf{B}\left[\left(1+\left.\frac{\partial f_{J}}{\partial \varepsilon_{J}}\right|_{\varepsilon_{J}=F_{J}}\right) \nabla \delta F_{J}^{\prime}+F_{J}^{\prime} \nabla\left(\left.\frac{\partial f_{J}}{\partial \varepsilon_{J}}\right|_{\varepsilon_{J}=F_{J}}\right)\right]\right] \\
& +\mathscr{H} \sum_{J} \nabla\left[\left(\delta F_{J}+\delta f_{J}\right) \nabla \Phi\right]=0 .
\end{aligned}
$$


Now, to compare some of terms above with the previously cancelled ones, we should remember that, first, all terms in (A.31) were divided by $\kappa a^{2}$ and second, the expression was acted on with $\Delta$. This means that either we should apply $\Delta$ to the cancelled terms or, vice versa, apply $\Delta^{-1}$ to the remaining ones. For example, the cancelled term $\propto \kappa a^{2} \mathscr{H}^{2} \sum_{J} \zeta_{J} \Rightarrow \mathscr{H}^{2} \sum_{J} \Delta \zeta_{J}=$ $\mathscr{H}^{2} \sum_{J} \nabla\left[\left(F_{J}+f_{J}\left(F_{J}\right)\right) \tilde{\mathbf{v}}_{J}\right] \sim \mathscr{H}^{2} \sum_{J} \nabla\left(F_{J} \tilde{\mathbf{v}}_{J}\right)$, where we have used (23). Then, employing the conditions $\delta F_{J} \sim \delta f_{J},\left|\partial f_{J} / \partial \varepsilon_{J}\right| \sim 1$ and taking into account that time derivatives with respect to $\eta$ are proportional in order to the Hubble parameter $\sim \mathscr{H}$, it can be deduced that all three terms in the second line behave as $\mathscr{H}^{2} \sum_{J} \nabla\left(\mathbf{B} \delta F_{J}\right)$. Thus, the ratio of these terms and the cancelled term is of the order of $\left(\delta F_{J} / F_{J}\right)\left(B / \tilde{v}_{J}\right) \sim B \tilde{v}_{J} / \Phi \sim \epsilon$ (see the estimate (18)). Similarly, the very last term $\propto \mathscr{H} \delta F_{J} \Phi$ may also be neglected provided that terms of higher order, i.e. $\propto \kappa a^{2} \mathscr{H} \sum_{J}\left(\delta F_{J}+\delta f_{J}\right)$ have already been cancelled in the above steps. Eventually, (A.31) is reduced to

$$
\begin{aligned}
\Delta & {\left[\Phi \sum _ { J } \left(\overline{\left.\frac{\partial f_{J}}{\partial \varepsilon_{J}}\right|_{\varepsilon_{J}=F_{J}}\left[-3 \mathscr{H}\left(F_{J}+f_{J}\left(F_{J}\right)\right)\right]}\right.\right.} \\
& +\left.3 \mathscr{H}\left(F_{J}+f_{J}\left(F_{J}\right)\right) \frac{\partial f_{J}}{\partial \varepsilon_{J}}\right|_{\varepsilon_{J}=F_{J}} \\
& \left.\left.-\left.3 \mathscr{H}\left(\delta F_{J}+\delta f_{J}\left(F_{J}\right)\right) \frac{\partial f_{J}}{\partial \varepsilon_{J}}\right|_{\varepsilon_{J}=F_{J}}\right)\right]=0
\end{aligned}
$$

and taking into consideration that $\left.\frac{\partial f_{J}}{\partial \varepsilon_{J}}\right|_{\varepsilon_{J}=F_{J}}$ is of the order of unity, we are left with the term $\propto \Phi \mathscr{H}\left(\delta F_{J}+\delta f_{J}\left(F_{J}\right)\right)$, which is again to be neglected in comparison to previously cancelled terms in the expression. Therefore, the LHS of this equation is equal to zero up to adopted accuracy, and this serves as the proof of the Eq. (A.24).

To conclude this appendix, it is worth noting that in a similar way we can also prove the relation $\mathbf{B}^{\prime}+2 \mathscr{H} \mathbf{B}=0$.

\section{References}

1. N. Aghanim et al. [Planck Collaboration], Planck 2018 results. VI. Cosmological parameters. A\&A 641, A6 (2020). https://doi.org/ 10.1051/0004-6361/201833910. arXiv:1807.06209 [astro-ph.CO]

2. B. Ratra, P.J.E. Peebles, Cosmological consequences of a rolling homogeneous scalar field. Phys. Rev. D. 37, 3406 (1988). https:// doi.org/10.1103/PhysRevD.37.3406

3. R.R. Caldwell, R. Dave, P.J. Steinhardt, Cosmological imprint of an energy component with general equation of state. Phys. Rev. Lett. 80, 1582 (1998). https://doi.org/10.1103/PhysRevLett. 80.1582. arXiv:astro-ph/9708069

4. J. Weller, A.M. Lewis, Large-scale cosmic microwave background anisotropies and dark energy. MNRAS 346, 987 (2003). https://doi. org/10.1111/j.1365-2966.2003.07144.x. arXiv:astro-ph/0307104
5. M. Bouhmadi-López, P.F. Gonzalez-Diaz, A. Zhuk, Topological defect brane-world models. Gravit. Cosmol. 8, 285 (2002). arXiv:hep-th/0207170

6. A. Zhuk, Integrable scalar field multi-dimensional cosmologies. Class. Quantum Gravity 13, 2163 (1996). https://doi.org/10.1088/ 0264-9381/13/8/012

7. A. Kamenshchik, U. Moschella, V. Pasquier, An alternative to quintessence. Phys. Lett. B 511, 265 (2001). https://doi.org/10. 1016/S0370-2693(01)00571-8. arXiv:gr-qc/0103004

8. N. Bilić, G.B. Tupper, R.D. Viollier, Unification of dark matter and dark energy: the inhomogeneous Chaplygin gas. Phys. Lett. B 535, 17 (2002). https://doi.org/10.1016/S0370-2693(02)01716-1. arXiv:astro-ph/0111325

9. M.C. Bento, O. Bertolami, A.A. Sen, Generalized Chaplygin gas, accelerated expansion, and dark-energy-matter unification. Phys. Rev. D 66, 043507 (2002). https://doi.org/10.1103/PhysRevD.66. 043507. arXiv:gr-qc/0202064

10. H.B. Benaoum, Accelerated Universe from modified Chaplygin gas and tachyonic fluid. arXiv:hep-th/0205140

11. J.M. Bardeen, Gauge-invariant cosmological perturbations. Phys. Rev. D. 22, 1882 (1980). https://doi.org/10.1103/PhysRevD.22. 1882

12. P.J.E. Peebles, The Large-Scale Structure of the Universe (Princeton University Press, Princeton, 1980)

13. V.F. Mukhanov, H.A. Feldman, R.H. Brandenberger, Theory of cosmological perturbations. Phys. Rep. 215, 203 (1992). https:// doi.org/10.1016/0370-1573(92)90044-Z

14. V.F. Mukhanov, Physical Foundations of Cosmology (Cambridge University Press, Cambridge, 2005)

15. R. Durrer, The Cosmic Microwave Background (Cambridge University Press, Cambridge, 2008)

16. D.S. Gorbunov, V.A. Rubakov, Introduction to the Theory of the Early Universe: Cosmological Perturbations and Inflationary Theory (World Scientific, Singapore, 2011)

17. M. Eingorn, First-order cosmological perturbations engendered by point-like masses. ApJ 825, 84 (2016). https://doi.org/10.3847/ 0004-637X/825/2/84. arXiv:1509.03835 [gr-qc]

18. M. Eingorn, C. Kiefer, A. Zhuk, Scalar and vector perturbations in a universe with discrete and continuous matter sources. JCAP 09, 032 (2016). https://doi.org/10.1088/1475-7516/2016/09/032. arXiv:1607.03394 [gr-qc]

19. M. Eingorn, C. Kiefer, A. Zhuk, Cosmic screening of the gravitational interaction. Int. J. Mod. Phys. D 26, 1743012 (2017). https:// doi.org/10.1142/S021827181743012X. arXiv:1711.01759 [gr-qc]

20. M. Eingorn, R. Brilenkov, Perfect fluids with $w=$ const as sources of scalar cosmological perturbations. Phys. Dark Univ. 17, 63 (2017). https://doi.org/10.1016/j.dark.2017.08.003. arXiv:1509.08181 [gr-qc]

21. R. Brilenkov, M. Eingorn, Second-order cosmological perturbations engendered by point-like masses. ApJ 845, 153 (2017). https://doi.org/10.3847/1538-4357/aa81cd. arXiv:1703.10282 [gr-qc]

22. M. Eingorn, N.D. Guran, A. Zhuk, Analytic expressions for the second-order scalar perturbations in the $\Lambda$ CDM Universe within the cosmic screening approach. Phys. Dark Univ. 26, 100329 (2019). https://doi.org/10.1016/j.dark.2019.100329. arXiv:1903.09024 [gr-qc]

23. M. Eingorn, A.E. Yükselci, A. Zhuk, Effect of the spatial curvature of the Universe on the form of the gravitational potential. Eur. Phys. J. C 79, 655 (2019). https://doi.org/10.1140/epjc/ s10052-019-7169-6. arXiv:1905.09502 [gr-qc]

24. Ö. Akarsu, R. Brilenkov, M. Eingorn, V. Shulga, A. Zhuk, Scalar perturbations in cosmological $f(R)$ models: the cosmic screening approach. Eur. Phys. J. C 78, 609 (2018). https://doi.org/10.1140/ epjc/s10052-018-6091-7. arXiv:1806.02669 [gr-qc] 
25. M. Eingorn, Cosmological law of universal gravitation. Int. J. Mod. Phys. D 26, 1750121 (2017). https://doi.org/10.1142/ S0218271817501218. arXiv:1709.02264 [gr-qc]

26. E. Canay, M. Eingorn, Duel of cosmological screening lengths. Phys. Dark Univ. 29, 100565 (2020). https://doi.org/10.1016/j. dark.2020.100565. arXiv:2002.00437 [gr-qc]

27. A. Burgazli, V. Shulga, A.E. Yükselci, A. Zhuk, Effect of peculiar velocities on the gravitational potential in cosmological models with perfect fluids. Phys. Lett. B 809, 135761 (2020). https://doi. org/10.1016/j.physletb.2020.135761. arXiv:2005.06237 [gr-qc]

28. V. Gorini, A.Y. Kamenshchik, U. Moschella, O.F. Piattella, A.A. Starobinsky, Gauge-invariant analysis of perturbations in Chaplygin gas unified models of dark matter and dark energy. JCAP 02, 016 (2008). https://doi.org/10.1088/1475-7516/2008/02/016. arXiv:0711.4242 [astro-ph]

29. J.C. Fabris, H.E.S. Velten, W. Zimdahl, Matter power spectrum for the generalized Chaplygin gas model: the relativistic case. Phys. Rev. D 81, 087303 (2010). https://doi.org/10.1103/PhysRevD.81. 087303. arXiv:1001.4101 [astro-ph.CO]

30. Y. Wang, D. Wands, L. Xu, J. De-Santiago, A. Hojjati, Cosmological constraints on a decomposed Chaplygin gas. Phys. Rev. D 87, 083503 (2013). https://doi.org/10.1103/PhysRevD.87.083503. arXiv:1301.5315 [astro-ph.CO]

31. P.P. Avelino, L.M.G. Beça, J.P.M. de Carvalho, C.J.A.P. Martins, E.J. Copeland, Onset of the nonlinear regime in unified dark matter models. Phys. Rev. D 69, 041301 (2004). https://doi.org/10.1103/ PhysRevD.69.041301. arXiv:astro-ph/0306493

32. P.P. Avelino, L.M.G. Beca, J.P.M. de Carvalho, C.J.A.P. Martins, The $\Lambda \mathrm{CDM}$ limit of the generalized Chaplygin gas scenario. JCAP 09, 002 (2003). https://doi.org/10.1088/1475-7516/2003/09/002. arXiv:astro-ph/0307427

33. P.P. Avelino, L.M.G. Beça, C.J.A.P. Martins, Linear and nonlinear instabilities in unified dark energy models. Phys. Rev. D 77, 063515 (2008). https://doi.org/10.1103/PhysRevD.77.063515. arXiv:0711.4288 [astro-ph]
34. R.A. Sussman, Quasilocal variables in spherical symmetry: numerical applications to dark matter and dark energy sources. Phys. Rev. D 79, 025009 (2009). https://doi.org/10.1103/PhysRevD.79. 025009. arXiv:0801.3324 [gr-qc]

35. A. Aviles, A. Bastarrachea-Almodovar, L. Campuzano, H. Quevedo, Extending the generalized Chaplygin gas model by using geometrothermodynamics. Phys. Rev. D 86, 063508 (2012). https://doi.org/10.1103/PhysRevD.86.063508. arXiv:1203.4637 [gr-qc]

36. M. Eingorn, A. Zhuk, Hubble flows and gravitational potentials in observable Universe. JCAP 09, 026 (2012). https://doi.org/10. 1088/1475-7516/2012/09/026. arXiv:1205.2384 [astro-ph.CO]

37. M. Eingorn, A. Zhuk, Remarks on mechanical approach to observable Universe. JCAP 05, 024 (2014). https://doi.org/10.1088/ 1475-7516/2014/05/024. arXiv:1309.4924 [astro-ph.CO]

38. N.E. Chisari, M. Zaldarriaga, Connection between Newtonian simulation and general relativity. Phys. Rev. D 83, 123505 (2011). https://doi.org/10.1103/PhysRevD.83.123505. arXiv:1101.3555 [astro-ph.CO]

39. D. Baumann, A. Nicolis, L. Senatore, M. Zaldarriaga, Cosmological non-linearities as an effective fluid. JCAP 07, 051 (2012). https://doi.org/10.1088/1475-7516/2012/07/051. arXiv:1004.2488 [astro-ph.CO] 